\title{
Merging Medical Imaging with Molecular Genetics for "Tailor-Made" Therapy in Oncology
}

\author{
Aikaterini Solomou*, Antonios Pikoulas and Vasileios Patriarcheas \\ Radiology Department, Greece
}

*Corresponding author: Aikaterini Solomou, Radiology Department, Greece

\begin{tabular}{|c|c|}
\hline ARTICLE INFO & ABSTRACT \\
\hline Received: 幽 April 20, 2019 & Merging medical imaging with molecular genetics, or simply radiogenomics, is an \\
\hline Published: 慧 April 26, 2019 & $\begin{array}{l}\text { emerging field which tries to correlate radiologic presentation of disease with its molecu- } \\
\text { lar characteristics (mutations and other genetic alterations). This correlation can lead to }\end{array}$ \\
\hline
\end{tabular}

P, Vasileios P. Merging Medical Imaging with Molecular Genetics for "Tailor-Made" Therapy in Oncology. Biomed J Sci \& Tech Res 17(3)-2019. BJSTR. MS.ID.003015.

\section{Introduction}

The genomic revolution has transformed our knowledge in both understanding disease mechanism and in the therapeutic strategies that are planned and followed [1]. During the last decades, therapeutics moved from the traditional treatment approach "one-size-fits-all" to precision medicine which aims to give patients a "tailor-made" therapy in order to achieve an adequate response as well as reduce possible complications. Precision medicine considers genetic variability between different individuals, environmental status as well as specific disease characteristics e.g. mutations [2]. Nowadays it is well understood that most human diseases are of great complexity and many times a disease's presentation/phenotype can be governed by totally different changes in molecular level. This biological variability explains the reason why using traditional therapeutic approach and treatment will only benefit a subset of the population of patients (Responders), while the rest of patients will not respond in the same extent (Non-responders) [2,3].

The application of contemporary molecular techniques in cancer research (e.g. DNA microarrays or DNA sequencing) gave rise to biomarkers that can predict patient's response to specific treatments (patient selection) as well as to targeted therapies where drugs interfere and block molecules which are crucial for tumor progression [4]. All these new therapeutic concepts require genotyping of tissue samples. In most cases, this is performed using many different interventional procedures. Apart from that multi regional sampling is required in order to achieve tumor heterogeneity, a phenomenon that could explain why even targeted therapies in some patients are often of limited duration [5].

\section{What it is and How it is Done}

Current clinical radiology practice relies on the interpretation of images via visual assessment, experience of individuals and only few qualitative imaging metrics. While this traditional approach is of great value and has served clinical practice for many decades, there is still need for the develop-ment of new radiological methods that would allow a more precise characterization and prediction of diseases in order to better assess the treatment response and prognosis toward the goal of personalized or precision medicine $[6,7]$. An effort to combine all the above was done quite recently leading to the creation of new concepts termed "radiomics", "radiogenomics" and "imaging genomics". Radiogenomics refers to the try of finding possible correlations between specific molecular characteristics of tumors (mutations or genetic alterations) and the radiologic presentation that these malignancies have in a variety of classic radiology methods (CT, MRI etc) [7]. In this way imaging biomarkers for specific genomic subtypes of tumors are being tested and screened as part of an effort to develop non-invasive genotyping methods.

The first step in the process is acquiring high quality images using typical radiological practices. This is done in everyday clinical actice and it is used for planning and diagnostic purposes. Using 
these images, the macroscopic definition of the tumor is done either in an automated way (using computational algorithms etc) or by an experience's physician (usually radiologist or radiology oncologist). Next step is the extraction of specific quantitative characteristics of the tumor: interaction and infiltration of tumor in surrounding areas and tissues, texture heterogeneity, different regions with different intensity levels, various factors describing size and shape etc. From these extracted data and features the most informative ones are selected (based on reproducibility, prominence and independence from other traits) and used to define if there are possible correlation between these traits and genomic features of tumor, clinical outcome and response to specific therapies [8].

\section{Limitations}

There are various challenges that radiogenomics must face and overcome. In fact, every step of the procedure has different limitations that all must be considered and overcome in order to increase the positive out-come of such an approach. One of the most important limitations that Radiogenomics face is the limited amount of data. That is because it is a novel field that requires time and effort from many different institutions in order to enrich available data and find hidden correlations that await to be elucidated. Another limitation is that multiple imaging acquisition is costly and time consuming. In order to overcome this, images are limited to the standard of care images taken in everyday clinical practice. The expectation is that eventually big data sets will be achieved, and this will have a more significant clinical impact [9].

Furthermore, different institutions use different parameters in imaging protocols (image resolution, washout period when using PET, patients' position) or even different algorithms and software in the reconstruction of images. Another difficulty is trying to identify and linking a large number of image data examples to similar clinical parameter such as disease stage [9]. Also, the manual segmentation of the tumor is though as ground truth. In fact, this step has high inter-reader variability and requires a serious amount of time when examining large data sets. For the radioge-nomics to have an important role in the future clinical practice large numbers of data sets are required. Overall the above makes it quite clear that a stand-ardized and automated method of tumor segmentation with minimal operator interaction and action is required.

\section{Advantages Against Typical Genotyping}

One could say that biopsy based genotyping has a clear benefit against radiogenomics. This should not be considered true as in classical genotyping samples from specific regions are used. The approach has changed, and multi-regional samples are now used in order to define tumour heterogeneity, but it remains an invasive practice that holds dangers. On the other hand, using imaging biomarkers via radiogenomics could allow similar results in a noninvasive way. Another advantage of radiogenomics is repetition. Imaging methods can be performed more than once and improve results while on the other hand most of the invasive methods have limitations about the total number of times allowed or the time intervals between two consecutive tries. Thirdly radiogenomics is cost effective allowing data being extract-ed from routine clinical imaging and being used in favour of the patient [10].

As radio genomics is a new field available data from studies in the literature is quite limited. Nevertheless, there is an increasing number of publications where science groups are trying to use radiogenomic methods to prove an existing correlation between the molecular characteristics of specific tumors and the radiologic image these tumor present. Most studies are using data from Glioblastoma, breast cancer and lung cancer [11-13].

\section{Conclusion}

Merging imaging with molecular genetics, can lead to a new, rapid and reliable tool in every day clinical practice for both diagnosis and prognosis. For this purpose, more research is needed to be done, in order to confirm correlation between imaging and gene expression profiling.

\section{References}

1. Hofker MH, FU J, Wijmenga C (2014) The genome revolution and its role in un-derstanding complex diseases. Biochim Biophys Acta 1842(10): 1889-1895.

2. Lu YF, Goldstein DB, Angrist M, Cavalleri G (2014) Personalized medicine and human genetic diversity. Cold Spring Harb Perspect Med 4(9): $\mathrm{a} 008581$.

3. Schork NJ (2015) Personalized medicine: Time for one-person trials. Nature 520(7549): 609-611.

4. Ow TJ, Sandulache VC, Skinner HD, Myers JN (2013) Integration of cancer ge-nomics with treatment selection: from the genome to predictive biomarkers. Cancer 119(22): 3914-3928.

5. Cortés JM, de Petris G, López JI (2017) Detection of Intratumor Heterogeneity in Modern Pathology: A Multisite Tumor Sampling Perspective. Front Med 4: 25.

6. Wu J, Tha KK, Xing L, Li R (2018) Radiomics and radiogenomics for precision radiotherapy. J Radiat Res 59(suppl_1): i25-i31.

7. Mazurowski MA (2015) Radiogenomics: what it is and why it is important. J Am Coll Radiol 12(8): 862-866.

8. Lambin P, Rios Velazquez E, Leijenaar R, Carvalho S, Van Stiphout RG, et al. (2012) Radiomics: extracting more information from medical images using advanced feature analysis. Eur J Cancer 48(4): 441-446.

9. Kumar V, Gu Y, Basu S (2012) Radiomics: the process and the challenges. Magn Reson Imaging 30(9): 1234-1248.

10. Jansen RW, Van Amstel P, Martens RM, Kooi IE, Wesseling P, et al. (2018) Non-invasive tumor genotyping using radiogenomic biomarkers, a systematic review and on-cology-wide pathway analysis. Oncotarget 9(28): 20134-20155.

11. Kickingereder P, Bonekamp D, Nowosielski M, Kratz A, Sill M, et al. (2016) Radiogenomics of Glioblastoma: Machine Learning-based Classification of Molecular Characteristics by Using Multiparametric and Multiregional MR Imaging Features. Radiology 281(3): 907-918.

12. Pinker K, Chin J, Melsaether AN, Morris EA, Moy L (2018) Precision Medicine and Radiogenomics in Breast Cancer: New Approaches toward Diagnosis and Treatment. Radiology 287(3): 732-747.

13. Vardhanabhuti V Kuo (2018) Lung Cancer Radiogenomics: The Increasing Value of Imaging in Personalized Management of Lung Cancer Patients. J Thorac Imaging 33(1): 17-25. 


\section{ISSN: 2574-1241}

DOI: 10.26717/BJSTR.2019.17.003015

Aikaterini Solomou. Biomed J Sci \& Tech Res

(c) This work is licensed under Creative

Submission Link: https://biomedres.us/submit-manuscript.php

$\begin{array}{ll}\text { BIOMEDICAL } & \text { Assets of Publishing with us } \\ \text { RESEARCHES } & \text { Global archiving of articles } \\ \text { - Immediate, unrestricted online access }\end{array}$

